\title{
Existence and Uniqueness of Almost Periodic Solution for A Class of Nonlinear Delay Integro-differential Equation
}

\author{
Zongyi Hou \\ School of Mathematics and Statistics, Hechi University, Guangxi, Yizhou 546300, P. R. China \\ hcxyhouzongyi@163.com
}

Keywords: Delay nonlinear system; Almost periodic solution; Existence; Lyapunov functional; Globally uniformly asymptotically stable.

\begin{abstract}
In this paper, we consider a general delay nonlinear system. The existence of almost periodic solution of the system is discussed under some relatively weaker conditions by means of constructing a class of Lyapunov functional and asymptotic almost periodic functions. Finally, some examples are given to support our theoretical analyses.
\end{abstract}

\section{Introduction}

By using the exponential dichotomy and fixed point theorem, Chen [1] discussed the existence problem of $\mathrm{T}$-periodic solutions for the periodic system

$$
x^{\prime}(t)=A(t, x(t)) x(t)+\int_{-\infty}^{t} C(t, s) x(s) d s+f(t, x(t-\tau))+b(t)
$$

Kato and Imai [2] considered the periodic and almost periodic solutions for the nonlinear system $x^{\prime}(t)=f(t, x)+e(t)$,

and obtained the existence and uniqueness of the solutions. Under the condition that the system

$$
x^{\prime}(t)=f(t, x)+\int_{-\infty}^{0} g(t, s, x(t+s), x(t)) d s+h\left(t, x_{t}\right),
$$

Has a bounded solution and time delay $|h(t, x)| \rightarrow 0$, Hamaya [3] established the existence of solutions.

For a nonlinear time-delay system, however, it is difficult to determine whether the system has a bounded solution. Furthermore, the condition $|h(t, x)| \rightarrow 0$ is also a strong restriction. In this paper, we consider the following system

$$
x^{\prime}(t)=f(t, x)+\int_{-\infty}^{t} C(t, s) x(s) d s+g\left(t, x_{t}\right)+e(t)
$$

Under some weaker conditions, the existence of almost periodic solutions of the system is established.

Throughout this paper, $x \in R^{n},\|$.$\| denotes a fixed norm, f(t, x)$ is about $\mathrm{t}$ to $\mathrm{t}, g(t, \varphi)$ is about $\mathrm{t}$ to $\varphi$ uniform almost periodic continuous function, $e(t)$ is an almost periodic continuous function of t, and $C(t, s)$ is an almost periodic continuous function of $(t, s)$, that is, for any $\varepsilon>0$ there exists $\tau>0$ such that $|C(t+\tau, s+\tau)-C(t, s)|<\varepsilon$.

\section{Existence of Almost Periodic Solutions}

Firstly, we introduce some Lemmas which will be used in the following discussion 
Lemma $1.1^{[3]}$ The functional [,]: $R^{n} \times R^{n} \rightarrow R$ defined by the $[x, y]=\lim _{h \rightarrow 0} \frac{\|x+h y\|-\|x\|}{h}$ has the following properties

$$
[x, y]=\inf _{h>0} \frac{\|x+h y\|-\|x\|}{h}
$$

(2) $|[x, y]| \leq\|y\|$;

(3) $[x, y+z] \leq[x, y]+[x, z]$;

(4) $D^{+}\|u(t)\|=\left[u(t), u^{\prime}(t)\right]$,

where $D^{+}\|u(t)\|$ denotes the upper-right derivative of $\|u(t)\|$

Lemma $1.2^{[4]}$ For almost periodic functions $p(t)$ and arbitrary $b \in R$ $\lim _{t-s \rightarrow \infty} \frac{1}{t-s} \int_{s}^{t} p(u+b) d u=\lim _{t-s \rightarrow \infty} \frac{1}{t-s} \int_{s}^{t} p(u) d u \quad$ Furthermore, if $\lim _{t-s \rightarrow \infty} \frac{1}{t-s} \int_{s}^{t} p(u) d u=-\gamma<0$, then for any $b \in R$ there exist positive constants $\alpha, \beta$ which are independent of $b$ such that $\exp \left(\int_{s}^{t} p(u+b) d u\right)=\beta \exp (-\alpha(t-s)), \quad(t>s)$.

Now, we give our results and the proof as follows

Theorem 2.1 Suppose the following conditions hold

(H2.1) There is an almost periodic continuous function $p(t)$ and a constant $\gamma>0$ satisfying $\lim _{t-s \rightarrow \infty} \frac{1}{t-s} \int_{s}^{t} p(u) d u=-\gamma$

(H2.2) For any $(t, x),(t, y) \in R \times R^{n}$ and any almost periodic function $p(t)$ satisfying condition (H2.1), $\left[x-y, f(t, x)-f(t, y)+\int_{-\infty}^{t} C(t, s) x(s) d s-\int_{-\infty}^{t} C(t, s) y(s) d s \leq p(t)\|x-y\| ;\right.$

(H2.5) $f(t, 0)=0$ and there exists a constant $L>0$ such that $\|e(t)+g(t, 0)\|<L$ Then the system (1.4) has a unique almost periodic solution

Proof. Consider the following system $x^{\prime}(t)=f(t, x)+\int_{-\infty}^{t} C(t, s) x(s) d s+e(t)$

Firstly, We will prove the system (2.5) has a bounded solution. Assuming that $x(t)$ is a solution of (2.5), then we have

$$
\begin{aligned}
D^{+}\|x(t)\| & =\left[x(t), x^{\prime}(t)\right] \\
& =\left[x(t), f(t, x)+\int_{-\infty}^{t} C(t, s) x(s) d s+e(t)\right] \\
& \leq\left[x(t), f(t, x)+\int_{-\infty}^{t} C(t, s) x(s) d s\right]+[x(t), e(t)] \\
& \leq p(t)\|x(t)\|+\|e(t)\| \leq p(t)\|x(t)\|+L .
\end{aligned}
$$

By solving the differential inequality, we get $\|x(t)\| \leq \beta\|x(0)\|+\frac{\beta L}{\alpha} \quad(t>0)$. 
For the sequence $\left\{t_{n}\right\}, t_{n} \rightarrow+\infty(n \rightarrow \infty)$, according to (2.7), we know that $\left\{x\left(t_{n}\right)\right\}$ is a bounded sequence. Using the properties of the almost periodic functions, as $n \rightarrow \infty$, we obtain $f\left(t+t_{n}, x\right) \rightarrow f(t, x) ; e\left(t+t_{n}\right) \rightarrow e(t) ; \int_{-\infty}^{t} C\left(t+t_{n}, s+t_{n}\right) x\left(s+t_{n}\right) d s \rightarrow \int_{-\infty}^{t} C(t, s) x(s) d s$.

Since $x\left(t+t_{n}\right) \quad$ is the solution for the system

$$
x^{\prime}\left(t+t_{n}\right)=f\left(t+t_{n}, x\left(t+t_{n}\right)\right)+\int_{-\infty}^{t} C\left(t+t_{n}, s+t_{n}\right) x\left(s+t_{n}\right) d s+e\left(t+t_{n}\right),
$$

Letting $n \rightarrow \infty$ we have $x^{\prime}\left(t+t_{n}\right) \rightarrow x^{* \prime}(t) \quad$ and $\quad x^{* \prime}(t)=f\left(t, x^{*}\right)+\int_{-\infty}^{t} C(t, s) x^{*}(s) d s+e(t)$,

where $x^{*}(t)$ is a solution for the system (2.5). On the other hand, we have

$\left\|x\left(t+t_{n}\right)\right\| \leq \beta\|x(0)\|+\frac{\beta L}{\alpha} \quad\left(t>-t_{n}\right)$

Letting $n \rightarrow \infty \quad$ we can obtain $\left\|x^{*}(t)\right\| \leq \beta\|x(0)\|+\frac{\beta L}{\alpha} \quad(t \in R)$,

which means the system (2.5) has a bounded solution $x^{*}(t)(t \in R)$.

Secondly, we will prove that the bounded solution of (2.5) is unique. Assuming that $y(t)$ is a solution of (2.5), then we have

$$
D^{+}\left\|x^{*}(t)-y(t)\right\| \leq p(t)\left\|x^{*}(t)-y(t)\right\|,
$$

furthermore,

$$
\left\|x^{*}(t)-y(t)\right\| \leq\left\|x^{*}(0)-y(0)\right\| \exp \left(\int_{0}^{t} p(\sigma) d \sigma\right) \leq \beta\left\|x^{*}(0)-y(0)\right\| \exp (-\alpha t) \rightarrow 0 \quad(t \rightarrow \infty)
$$

Hence, the bounded solution is unique.

Thirdly, we will prove that the bounded sequence $\left\{x\left(t+t_{n}\right)\right\}$ is uniform convergent. For

$D^{+}\left\|x\left(t+t_{k}\right)-x\left(t+t_{m}\right)\right\| \leq p(t)\left\|x\left(t+t_{k}\right)-x\left(t+t_{m}\right)\right\|+\left\|e\left(t+t_{k}\right)-e\left(t+t_{m}\right)\right\|, \quad$ We have

$$
\left\|x\left(t+t_{k}\right)-x\left(t+t_{m}\right)\right\| \leq \beta\left\|x\left(t_{k}\right)-x\left(t_{m}\right)\right\|+\frac{\beta}{\alpha}\left\|e\left(t+t_{k}\right)-e\left(t+t_{m}\right)\right\| .
$$

Noticing that $\left\{x\left(t_{n}\right)\right\}$ is a bounded sequence, hence, for all $\varepsilon>0$ there is a sufficiently large positive $N_{1}$ such that $\left\|x\left(t_{k}\right)-x\left(t_{m}\right)\right\|<\frac{\varepsilon}{3 \beta}$ When $k, m \geq N_{1}$, Since $e(t)$ is an almost periodic continuous function, there exists a positive number $N_{2}$ such that $\left\|e\left(t+t_{k}\right)-e\left(t+t_{m}\right)\right\|<\frac{\alpha \varepsilon}{3 \beta}$ when $k, m \geq N_{2}$. Let $N_{0}=\max \left\{N_{1}, N_{2}\right\}$, then we get $\left\|x\left(t+t_{k}\right)-x\left(t+t_{m}\right)\right\|<\frac{\varepsilon}{3}+\frac{\varepsilon}{3}<\varepsilon$ when $k, m \geq N_{0}$ which shows that $\left\{x\left(t+t_{n}\right)\right\}$ is uniform convergent.

Finally, we obtain that $x(t)$ is an asymptotically almost periodic solution of $(2.5)$. From [5,10], there exists an almost periodic solution for the system (2.5). Since almost periodic functions are bounded and there is a unique bounded solution for the system (2.5), we can infer that there is a unique almost periodic solution for the system (2.5).

Defining a set $B=\{u(t): u(t)$ is an almost periodic function $\}$, then $\mathrm{B}$ is a Banach space under the norm $\|u(t)\|=\sup _{t \in R}|u(t)|$ For any $u(t) \in B$, 
Consider the function $x_{u}(t)=f(t, x)+\int_{-\infty}^{t} C(t, s) x(s) d s+g\left(t, x_{t}\right)+e(t)$.

(2.13) Define the

operator $\mathrm{T}:{ }^{u(t) \rightarrow x_{u}(t)}$. Under the conditions of the theorem, we need to prove that there exists a subset of $B_{0}$ such that $B_{0} \rightarrow B_{0}$ is a completely continuous operator.

Denoting $B_{n}=\{u(t): u(t) \in B$ and $\|u(t)\| \leq n\}$, where $\mathrm{n}$ is a natural number. Then we can conclude that there exists a natural number $\mathrm{N}$ such that $T: B_{N} \rightarrow B_{N}$. If not, there is $u_{n} \in B_{n}$ for any natural number $\mathrm{n}$ such that $\left\|T u_{n}\right\|>n$. By condition (H2.4), there is a natural number $N \geq \max \left\{4 \beta\|u(0)\|, \frac{4 \beta L}{\alpha}\right\}$ for $\varepsilon \leq \frac{1}{4}$, when $n \geq N$ then we have $\frac{1}{n} \sup _{\|\varphi\| \leq n}\|g(t, \varphi)\| \leq \varepsilon \leq \frac{1}{4}$ According to (2.7), (2.13), we can obtain $\frac{1}{n}\left\|T u_{n}(t)\right\| \leq \frac{\beta}{n}\left\|u_{n}(0)\right\|+\frac{\beta L}{n \alpha}+\frac{1}{n} \sup _{\|\varphi\| \leq n}\left\|g\left(t, \varphi_{n}\right)\right\| \leq \frac{3}{4}$. This conflicts with $\left\|T u_{n}\right\|>n$ Hence, there is a natural number $\mathrm{N}$ such that $T: B_{N} \rightarrow B_{N}$.

Next, we will prove that the $T B_{N}$ is a compact subset in B. Since $T B_{N} \subseteq B_{N},\left\{T u(t) ; u(t) \in B_{N}\right\}$ is uniformly bounded. Denote $A_{1}=\sup _{t \in R}\left\{T u(t) ; u(t) \in B_{N}\right\}$ Using the assumptions, $f(t, x)$ is the uniform almost periodic continuous function about $\mathrm{t}$ for $\mathrm{x}$, then we can obtain that $f(t, u)$ is bounded when $\|u(t)\| \leq N$ According to $\frac{1}{n} \sup _{\|\varphi\| \leq n}\|g(t, \varphi)\| \rightarrow 0(n \rightarrow \infty)$, we can infer that $\|g(t, \varphi)\|$ is bounded.

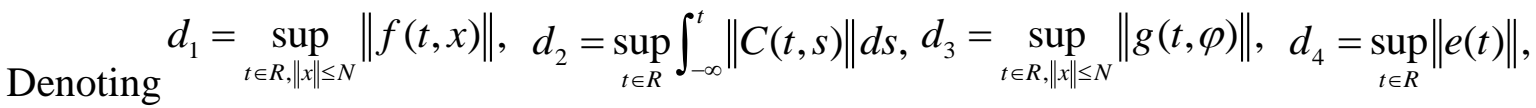

We have

$$
\frac{d T u(t)}{d t}=\frac{d x_{n}}{d t}=f\left(t, x_{n}(t)\right)+\int_{-\infty}^{t} C(t, s) x_{u}(s) d s+g\left(t, x_{u_{t}}(t)\right)+e(t)
$$
$\left\|\frac{d T u(t)}{d t}\right\| \leq d_{1}+d_{2} N+d_{3}+d_{4}$.

Hence, $\left\{T u(t) ; u(t) \in B_{N}\right\}$ is uniformly bounded and equicontinuous.

So by Ascoli theorem, $T B_{N}$ is a compact subset of $B_{N}$

At last, we will prove $T$ is continuous in $B_{N}$ For any $\varepsilon>0$, by the condition (H2.1), there is sufficiently large $K>0$ such that $\exp \left(\int_{t-K}^{t} p(s) d s\right)<\frac{\varepsilon}{4 A_{1}}$.Denoting $A_{2}=\int_{t-K}^{t} \exp \left(\int_{s}^{t} p(\sigma) d \sigma\right) d s$, we know that $\mathrm{f}$ and $\mathrm{g}$ are continuous functions according to the assumptions. Hence, for any

$$
\begin{aligned}
u(t), v(t) \in B_{N}, \text { we have } & \left\|g\left(t, u_{t}\right)-g\left(t, v_{t}\right)\right\|<\frac{\varepsilon}{2 A_{2}},{ }_{\text {when }}\left\|u_{t}-v_{t}\right\|<\delta(\varepsilon) \text {.For } \\
D^{+}\|T u(t)-T v(t)\| & \leq p(t)\|T u(t)-T v(t)\|+\left\|g\left(t, u_{t}\right)-g\left(t, v_{t}\right)\right\|, \text { we can obtain } \\
\|T u(t)-T v(t)\| & \leq\|T u(t-K)-T v(t-K)\| \exp \left(\int_{t-K}^{t} p(s) d s\right. \\
& +\left\|g\left(t, u_{t}\right)-g\left(t, v_{t}\right)\right\| \int_{t-K}^{t} \exp \left(\int_{s}^{t} p(\sigma) d \sigma\right) d s(2.16) \\
& <2 A_{1} \cdot \frac{\varepsilon}{4 A_{1}}+\frac{\varepsilon}{2 A_{2}} \cdot A_{2}=\varepsilon,
\end{aligned}
$$

furthermore, 
Which means that $\mathrm{T}$ is continuous in $B_{N}$ Therefore, $\mathrm{T}$ is completely continuous in $B_{N}$.

According to Schrader fixed point theorem, $\mathrm{T}$ has a fixed point in ${ }^{B_{N}}$, that is, there is an almost periodic solution for (1.4).

Obviously, this is the unique almost periodic solution.

\section{Conclusions}

In this paper, we have discussed the existence of almost periodic solution for a class of nonlinear delay integro-differential equations under some relatively weaker conditions by means of constructing a class of Lyapunov functional and asymptotic almost periodic functions. Some examples are given to support our theoretical analyses.

\section{Acknowledgement}

It is a project supported supported by Scientifics Research Foundation of the Education Department of Guangxi Zhuang Autonomous Region (no. KY2015ZD103) .The corresponding author is Zongyi Hou.

\section{References}

[1] F. D. Chen. Acta Mathematicae Applicatae Sinica, 2003, 26(1): 141-148.

[2] S. Kato, M. Imai. Nonlinear Analysis, 1995, 24: 1183-1192.

[3] Y. Hamaya. 1989, 41(2): 105-116.

[4] Q. Y. Wang. Acta Mathematica Sinica, 1997, 40: 80-89.

[5] A. M. Fink. Lecture Notes in Mathematics. Springer-Verlag, 1974.

[6] T. Yoshizawa. New York: Springer- Verlag, 1975.

[7] H.J.Xiang,J.D.Cao. Nonlinear Analysis 2009,71:6097-6108

[8] A.M.Fink. Lecture Notes in Mathematics.Springer-Verlag,1974.

[9] X.X.Chen,F.X.Lin. Nonlinear Analysis 2010,11(2):1182-1189.

[10] H.Gao,B.W.Liu. Applied Mathematical Modelling,2010,34(1):72-79. 